\section{El clima organizacional y su influencia en el rendimiento laboral de los trabajadores de la empresa G.S.H S.A.}

\author{
The climate organizational and its influence in the \\ performance of workers at company GSH S.A.
}

\begin{abstract}
RESUMEN
Los estudios sobre clima organizacional comenzaron a tener relevancia a causa de la formación de grupo como organización, atribuyéndole causas externas e internas por un lado con efectos negativos y positivos por otro lado, siendo este último, un elemento imprescindible para que el trabajador se sienta motivado y apoyado por las personas de su entorno, trayendo como consecuencia un mayor rendimiento, rentabilidad y productividad en los miembros que conforman la organización. Este constructo ha sido relacionado directamente con el rendimiento del grupo, puesto que ambos conceptos son controlados por el trabajador e impulsados, de manera positiva o negativa, por los gerentes, personal de cargo superior y la cultura de la organización. Por ello, es necesario comprender la relación directa que existe entre ambos conceptos, y entender la necesidad intrínseca por parte de todos miembros de la organización respecto al logro de objetivos comunes trazados tanto por el trabajador como por la organización. Sin embargo, en el contexto de las empresas familiares estos conceptos son desconocidos o simplemente dan la apariencia de ser por aceptados por los trabajadores, de modo tal, que el dueño o gerente de la empresa, no se preocupa por motivar o incentivar al personal y por ende brindar un mejor ambiente laboral dentro de su empresa.
\end{abstract}

Palabras claves: Clima organizacional; rendimiento laboral; objetivos.

\begin{abstract}
Studies on organizational climate began to have relevance because of the formation of a group as an organization, attributing external and internal causes on the one hand with negative and positive effects on the other hand, the latter being an essential element for the worker to feel motivated and supported by the people around him, resulting in higher performance, profitability and productivity in the members that make up the organization. This construct has been directly related to group performance, since both concepts are controlled by the worker and driven, positively or negatively, by managers, senior staff and the culture of the organization. However, in the context of family businesses these concepts are unknown or
\end{abstract}

Olga Liz Carhuayal Huaranga

olga.carhuayal@unmsm.edu.pe Universidad Nacional Mayor de San Marcos, Facultad de Ciencias Administrativas. Lima, Perú 
simply give the appearance of being accepted by the workers, in such a way that the owner or manager of the company does not care about motivating or encouraging the staff and therefore provide a better work environment within your company.

Keywords: Organizational climate; work performance; objectives.

\section{INTRODUCCIÓN}

Frente a los constantes cambios, tecnológicos, sociales, económicos, etc., que afectan a las diversas industrias, las organizaciones se ven en la necesidad de aplicar cambios en su estructura empresarial, lo que llevará al desarrollo de la gestión empresarial y al éxito de sus objetivos. Esto se debe a que la gestión empresarial funciona con el apoyo de personas, por lo que busca obtener un mayor rendimiento, rentabilidad y productividad entre los miembros que conforman la organización.

Sin embargo, estos cambios se ven afectados cuando se habla de empresas familiares, puesto que, en este tipo de organizaciones, las actividades de trabajo se realizan de manera empírica, sin conocimiento previo de los procesos, por un lado; y desconocimiento de la importancia de laboral en un adecuado clima organizacional por otro lado.

Por lo tanto, el objetivo del presente artículo científico es conocer la relación que existe entre el clima organizacional y el rendimiento laboral de los trabajadores que laboran dentro de una empresa familiar, donde los dueños de las empresas tienen algún tipo de vínculo familiar con los demás trabajadores.

\section{Clima Organizacional.}

El concepto de ambiente laboral o clima organizacional fue dado a conocer, inicialmente, en el área de psicología industrial por Gellerman en el año 1960. Gellerman (1960: citado en Brunet, 1987) señalaba que el "concepto de clima organizacional se encontraba conformado por una amalgama de dos escuelas de pensamiento: la escuela Gestalt y la escuela funcionalista" (p. 14).

La primera orientó este concepto hacia la percepción y se basó en dos principios, captar el orden de las cosas tal como se presentan y el de la creación de un nuevo orden, es decir, el entorno influye en el comportamiento del trabajador. En cambio, la escuela funcionalista señala al pensamiento y al comportamiento como base central del clima organizacional, e indica que la persona no tiene otra opción que adaptarse al medio y participar en la determinación del clima (Gutiérrez, 2001).

Asimismo, Pelaes (2010) afirmó lo siguiente: "el clima organizacional puede ser la característica o propiedad del ambiente laboral, que perciben o experimentan los integrantes de la organización, y que ejerce influencia en su conducta laboral" (p. 46). De igual manera, Jahuira (2015) indicó que el clima organizacional es "percepción que comparten los integrantes de una organización sobre el mismo trabajo, el entorno físico en que desarrollan su labor, las relaciones interpersonales que se llevan a cabo en el trabajo y las diversas normas formales que lo afectan" (p. 20). Por otro lado, Huamaní (2015) señaló que el ambiente laboral es la interrelación entre grupos de personas y estructuras sean estas formales e informales dentro de una organización, lo cual puede influenciar de manera directa en el desempeño de los trabajadores.

El desarrollo de la gestión de talento humano dentro de las organizaciones ha tomado un impulso enorme. Esto es, porque las organizaciones se han dado cuenta de la importancia de brindar a sus trabajadores un lugar adecuado y agradable de trabajo, además de administrar correctamente los recursos de la empresa, lo cual no solo beneficia directamente al rendimiento del trabajador, sino también a las organizaciones.

\section{Enfoques del clima organizacional.}

Como consecuencia de los diversos estudios acerca del clima organizacional, se llegó a desarrollar tres enfoques principales que sustentan a este constructo: 
Tabla 1

Principales enfoques del clima organizacional

\begin{tabular}{ll}
\multicolumn{1}{c}{ Enfoque } & \multicolumn{1}{c}{ Autor(es) } \\
Estructuralista & Forehand y Gilmer \\
Subjetivo & Halpin y Crofts \\
Síntesis & Litwin y Stringer \\
\hline
\end{tabular}

Fuente: Elaboración propia

a. Enfoque Estructuralista, desarrollado por Forehand y Gilmer (1964) donde indican que el ambiente laboral es un conjunto de características propias de cada organización que se mantienen a largo plazo y que influyen en el comportamiento de los trabajadores. Al influir en el comportamiento de los trabajadores, se hace entonces indispensable que este último perciba el interés de la organización en su persona, y no solo como un activo para la organización.

b. Enfoque Subjetivo, representado por Halpin y Crofts (1963), señalan que el clima organizacional es la opinión que se forman los trabajadores acerca de la organización.

c. Enfoque de Síntesis, en este enfoque Litwin y Stringer (1968) unificaron los dos enfoques precedentes.

Así mismo, en este último enfoque realizado, Litwin y Stringer (1981) señalan la importancia de la motivación y el clima organizacional, por lo que "lograron el desarrollo de un modelo que permite describir los principales aspectos del comportamiento de las personas dentro de las organizaciones, considerando el clima y la motivación" (Delgado \& Adla, 2017, p. 55).

\section{Enfoque de estudio del Clima Organizacional.}

Debido a la trascendencia del enfoque de Síntesis, y mejor ajuste de esta teoría sobre la población de estudio de la empresa GSH S.A. se ha tomado como referencia el modelo de clima organizacional Litwin y Stringer

En el modelo desarrollado por estos autores, se pudo observar que la percepción del ambiente conduce a factores que motivan al trabajador a desarrollar un mayor rendimiento en sus tareas laborales, productividad, innovación, entre otros, lo cual se traduce no solo en un beneficio para la persona, sino también para la organización, entre ellos, una mayor productividad, menos rotación, etc.

Para poder realizar la medición del clima organizacional, Litwin y Stringer (1978), consideraron nueve dimensiones a saber:

Estructura: Representa el sentimiento de los empleados respecto a los controles, trámites, procedimientos, número de reglas, limitaciones entre otros, a la cual son sometidos sus funciones y trabajos, dentro de las organizaciones para la que trabajan.

Responsabilidad: Se refiere a la sensación que los empleados tienen de ser sus propios jefes al momento de realizar sus trabajos, es decir, que las decisiones son asumidas por el propio trabajador.

Recompensa: Representa a la sensación de los trabajadores respecto a un sistema adecuado o no de recompensas, lo cual es comparado de manera inmediata con sus pares.

Desafío: Corresponde a los retos que reciben los empleados durante el desarrollo de sus labores. Estos desafíos son desarrollados a fin de lograr las metas trazadas por la organización.

Relaciones: Es la percepción por parte de los empleados de la existencia de un ambiente laboral agradable, armonioso, de compañerismo y confraternidad entre todas las personas que trabajan dentro de la organización, dejando de lado las diferencias por temas de jerarquía.

Cooperación: Representa a la percepción de apoyo mutuo entre todos y cada uno de los trabajadores de la organización para poder enfrentar posibles conflictos de manera conjunta.

Estándares: Representa la percepción de los empleados sobre las normas y reglamentos que exige la organización sobre el rendimiento laboral.

Conflictos: Representa la capacidad de los trabajadores, tanto pares como superiores, para saber discrepar de manera adecuada, frente a las diferentes opiniones.

Identidad: Es el sentimiento de pertenecer a una organización en sí, donde es considerado un elemento importante y valioso dentro del grupo de trabajo. En este punto, los objetivo, 
tanto de la organización como del trabajador encuentran un sentido en común.

\section{Rendimiento Laboral.}

La definición multidimensional define al rendimiento laboral como determinados comportamientos que son importantes para el logro de metas, instituciones u objetivos de la organización, los cuales se encuentran controlados por el trabajador (Koopmans et al., 2016).

Este constructo trae a colisión la motivación que, para Kossen (1995) fue definida como "los impulsos diferentes internos o las fuerzas ambientales alrededor, que estimulan a los individuos a comportarse de una forma específica, motivar es generar el desplazamiento de una actitud hacia otra o de un comportamiento hacia otro distinto" (p. 345).

Por otro lado, una definición más actual:

El rendimiento laboral es uno de los constructos más explorados en la literatura de la psicología organizacional. Sin embargo, la historia de la disciplina hasta finales de la década del 80 se encuentra plagada de estudios destinados a evaluar de forma indiferenciada del rendimiento laboral, la productividad y la eficacia de los trabajadores. (Gabini, 2018, p. 27)

\section{Enfoques del rendimiento laboral.}

Debido a los diversos estudios y autores que buscaron desarrollar un modelo que represente la realidad del rendimiento laboral en las organizaciones, se llegaron a conocer varios paradigmas sobre este constructo, sin embargo, solo indicaremos los enfoques que se relacionan directamente con el contexto de la población de estudio (Ver Tabla 2).

\section{Enfoque de estudio del Clima Organizacional.}

De todos estos paradigmas, el más acertado y cercano a la realidad de la población de estudio fue la desarrollada por Koopmans et al. (2011) quienes agrupan las dimensiones mencionadas en los demás modelos dentro de tres grandes grupos, los cuales se describirán a continuación.

Rendimiento en las tareas: Esta dimensión está relacionada con dos características: primero, corresponde a la dificultad en las labores, la suficiencia y expertiz al momento de llevar a cabo sus funciones, y el estado de ánimo del trabajador; segundo, se basa en la situación física de su entorno, es decir; con qué facilidades cuenta el trabajador para realizar cómodamente sus actividades.

Rendimiento en el contexto: Son actividades relacionadas a la empresa, que se encuentran enfocados a mantener el ambiente laboral e interpersonal adecuados. Es decir, son comportamientos que desarrollan la cooperación entre los trabajadores lo cual tienen consecuencias positivas para la organización, cabe mencionar que este tipo de comportamiento son expresado de manera espontánea y no son remunerados.

Comportamientos laborales contraproducentes: Son aquellos comportamientos que perjudican de manera directa e indirecta a

Tabla 2

Principales enfoques del Rendimiento Laboral

\begin{tabular}{ll} 
Autores & Enfoques \\
\hline Borman, Motowidlo, Rose y Hansen (1985) & $\begin{array}{l}\text { Desarrollaron un modelo que considera cuatro dimensiones: } \\
\text { (a) rendimiento en la tarea, (b) lealtad, (c) trabajo en equipo, (d) determinación. }\end{array}$ \\
Murphy (1990) & $\begin{array}{l}\text { Las dimensiones del rendimiento laboral fueron las siguientes: (a) conductas orientadas } \\
\text { a las tareas laborales, (b) conductas interpersonales, (c) conductas relacionadas con la } \\
\text { pérdida de tiempo, (d) conductas destructivas y azarosas. }\end{array}$ \\
Borman y Motowidlo (1993) & $\begin{array}{l}\text { Sugirieron un nuevo modelo de dimensiones que agrupa los dos primeros modelos en } \\
\text { dos grandes factores: (a) relacionados a la tarea, (b) relacionados al contexto. }\end{array}$ \\
Koopmans et al. (2011) & $\begin{array}{l}\text { Indicaron que solo existen 3 dimensiones: (a) relacionado al rendimiento de las tareas } \\
\text { laborales, (b) Relacionado al contexto, (c) comportamiento contraproducente. }\end{array}$ \\
Al-Makhaita, Ahmed y Hafez (2014) & $\begin{array}{l}\text { Identificaron seis dimensiones: (a) liderazgo, (b) cuidado crítico, (c) enseñanza y/o } \\
\text { colaboración, (d) planeamiento y/o evaluación, (e) relaciones interpersonales y/o } \\
\text { comunicación y (f) desarrollo profesional. }\end{array}$ \\
\hline
\end{tabular}

Fuente: Elaboración propia. 
la efectividad organizacional. Tales como: la procrastinación, los comentarios mal intencionados, comportamientos destructivos, entre otros. Como consecuencia de estos tipos de comportamientos, los directivos de las organizaciones buscan promover un rendimiento mayor al promedio normal, en la realización de las labores, a través de un adecuado clima laboral.

\section{Empresa Familiar.}

Este tipo de empresas son muy comunes hoy en día, y forman parte importante de las actividades económicas del país, llegando a ser uno de los motores de desarrollo más grandes del Perú, sin embargo, la informalidad es enorme, por lo que no se cuenta con una cantidad precisa de estas empresas. "Bajo la denominación de la empresa familiar se agrupan aquellas compañías en las que una o varias familias tienen en sus manos grandes decisiones que pueden condicionar el futuro de la empresa" (Tapies, 2016, p. 7).

\section{Tipos de empresa familiar.}

Según Tapies (2016), las empresas familiares se clasifican en:

Inversión familiar: la inversión es familiar.

Trabajo familiar: donde se promueve que la mayoría o casi todo el personal de la empresa, tenga algún vínculo familiar.

Dirección familiar: donde solo las personas de la familia mejor capacitados ocupen puestos relevantes

Gobierno familiar: los miembros de la familia no participan en la dirección o gestión de la empresa.

Centrándonos en la población de estudio, el tipo de empresa corresponde al trabajo familiar.

Así mismo, se conoce que estas empresas no tienen un ciclo de vida prolongado, esto se debe, a que, en su mayoría, no cuenta con un plan estratégico o con una visión, misión que se proyecte al futuro o con un plan de contingencia que pueda sustentarlo a lo largo del desarrollo de sus actividades, sumado a la falta de personal idóneo para el desarrollo de determinadas actividades.

\section{MÉTODOS}

El artículo científico que se presenta tiene un enfoque cuantitativo de tipo correlacional, basados en la investigación realizada durante el año 2019, cuya selección de muestra es no probabilística, se estableció que el diseño de investigación pertenece al tipo transeccional.

Así mismo, se consideró como variable independiente el clima laboral y como variable dependiente, rendimiento laboral. No se manipuló ninguna de las dos variables por lo que se puede observar el fenómeno tal cual se presentó.

La población de estudio estuvo constituida por los 25 trabajadores de la empresa familiar G.S.H, para efectos del trabajo de investigación no se extraerá una muestra y esta será tipificada tipo censo, es decir, se considerará como tamaño de la muestra la misma cantidad de la población de estudio. Los métodos de investigación que se usaron fueron la encuesta, entrevista y la observación. Para la realización de la encuesta se contó con la participación del total de trabajadores.

\section{RESULTADOS}

El resultado obtenido en base a los métodos de investigación en relación al objetivo general de estudio:

- Determinar la relación entre clima organización y rendimiento laboral de los trabajadores de la empresa GSH S.A. Dentro del análisis de la muestra de estudio, indica que el $98 \%$ de los encuestados consideran que si existe una relación directa y proporcional entre las variables, clima organizacional y rendimiento

Así mismo, los resultados en relación a los demás objetivos específicos fueron:

1. Identificar la relación entre la dimensión estructura y las dimensiones del rendimiento laboral de los trabajadores de la empresa GSH S.A. De los resultados obtenidos el $80 \%$ de los encuestados identifica que existe una relación directa entre la estructura de la empresa y las dimensiones del rendimiento laboral. Por otro lado, el $20 \%$ de encuestados señaló que 
no percibe ninguna relación entre esas dos variables.

2. Identificar la relación entre la dimensión responsabilidad y las dimensiones del rendimiento laboral de los trabajadores de la empresa GSH S.A. De los resultados obtenidos el $87 \%$ de los encuestados identifica que existe una relación directa entre la responsabilidad y las dimensiones del rendimiento laboral. Por otro lado, el 13\% de encuestados señaló que no percibe ninguna relación entre esas dos variables.

3. Identificar la relación entre la dimensión recompensa y las dimensiones del rendimiento laboral de los trabajadores de la empresa GSH S.A. De los resultados obtenidos el 99\% de los encuestados identifica que existe una relación directa entre la recompensa y las dimensiones del rendimiento laboral. Por otro lado, el $1 \%$ de encuestados señaló que no percibe ninguna relación entre esas dos variables.

4. Identificar la relación entre la dimensión desafío y las dimensiones del rendimiento laboral de los trabajadores de la empresa GSH S.A. De los resultados obtenidos el $76 \%$ de los encuestados identifica que existe una relación directa entre el desafío y las dimensiones del rendimiento laboral. Por otro lado, el $24 \%$ de encuestados señaló que no percibe ninguna relación entre esas dos variables.

Identificar la relación entre la dimensión relaciones y las dimensiones del rendimiento laboral de los trabajadores de la empresa GSH S.A. De los resultados obtenidos el $70 \%$ de los encuestados identifica que existe una relación directa entre las relaciones y las dimensiones del rendimiento laboral. Por otro lado, el 30\% de encuestados señaló que no percibe ninguna relación entre esas dos variables.

5. Identificar la relación entre la dimensión cooperación y las dimensiones del rendimiento laboral de los trabajadores de la empresa GSH S.A. De los resultados obtenidos el $88 \%$ de los encuestados identifica que existe una relación directa entre la cooperación y las dimensiones del rendimiento laboral. Por otro lado, el $12 \%$ de encuestados señaló que no percibe ninguna relación entre esas dos variables.

6. Identificar la relación entre la dimensión estándares y las dimensiones del rendimiento laboral de los trabajadores de la empresa GSH S.A. De los resultados obtenidos el $56 \%$ de los encuestados identifica que existe una relación directa entre los estándares y las dimensiones del rendimiento laboral. Por otro lado, el $44 \%$ de encuestados señaló que no percibe ninguna relación entre esas dos variables.

7. Identificar la relación entre la dimensión conflictos y las dimensiones del rendimiento laboral de los trabajadores de la empresa GSH S.A. De los resultados obtenidos el $96 \%$ de los encuestados identifica que existe una relación directa entre los conflictos y las dimensiones del rendimiento laboral. Por otro lado, el $4 \%$ de encuestados señaló que no percibe ninguna relación entre esas dos variables.

8. Identificar la relación entre la dimensión identidad y las dimensiones del rendimiento laboral de los trabajadores de la empresa GSH S.A.De los resultados obtenidos el $71 \%$ de los encuestados identifica que existe una relación directa entre la identidad y las dimensiones del rendimiento laboral. Por otro lado, el 29\% de encuestados señaló que no percibe ninguna relación entre esas dos variables.

\section{DISCUSIÓN}

Para efectos del presente trabajo de investigación, se revisaron más de treinta antecedentes nacionales e internacionales para ambas variables, de los cuales solo seleccionaremos dos trabajos, los cuales se adaptan mejor a la población de estudio, estos trabajos respaldan y soportan la relación directa entre clima organizacional y rendimiento laboral.

Por un lado, Palacios (2019) demostró en su trabajo de investigación que las variables: clima organizacional y desempeño laboral del personal administrativo de los Distritos de Salud Pública de la provincia de Manabí, se relacionan de 
manera directa, esto es; al incentivar que cada trabajador busque su autorrealización; esto aumentaría la confianza en ellos mismos, además de lograr una mayor productividad e involucramiento dentro de su centro de labores, lográndose de manera eficiente cuando existe una comunicación adecuada entre los mismo trabajadores Así mismo, se llegó a la conclusión de que, a un mayor nivel de compromiso y participación por parte del personal, el nivel de conocimiento sobre los temas relacionados con sus labores, es mayor.

Y, por otro lado, Gabini (2018) centró su trabajo de investigación acerca del rendimiento laboral, dicho trabajo de investigación fue denominado "Potenciales predictores del rendimiento laboral: Una exploración empírica", el objetivo de este trabajo fue desarrollar y verificar un modelo explicativo de esta variable, considerando aspecto sociodemográficas, organizacionales y disposicionales.

En ambos trabajos de investigación las variables de estudio, clima organizacional y rendimiento laboral se encontraron acorde a las definiciones citadas por las diversas teorías, tanto por teorías desarrolladas en un principio como por las teorías actuales que complementan las primeras. Por lo que se puede confirmar en estos trabajos, la búsqueda continua y mejorada del capital humano es un factor relevante que, a pesar de los años, siempre seguirá siendo un tema de estudio, con lo cual los empresarios buscan aumentar la productividad de sus trabajadores y, por lo tanto, las utilidades de sus empresas.

\section{CONCLUSIONES}

Por todo lo antes expuesto, se puede llegar a la siguiente conclusión general, que, si existe una relación directa entre ambas variables, clima organizacional y rendimiento laboral, toda vez que de la bibliografía revisada sustento de manera absoluta dicho comportamiento.

- Los factores de estructura como controles, trámites, procedimientos, reglas, entre otros se relacionan de manera directa al rendimiento del trabajador, puesto que estos factores si son positivos o de agrado respecto al trabajador, estos lo verán de manera agradable.
- La responsabilidad percibida por parte de los jefes afecta de manera directa al rendimiento de los trabajadores, así mismo la responsabilidad en el desarrollo de sus labores respecto a sus pares o compañeros.

- El factor recompensa resultó indispensable para un mayor compromiso con las actividades de la empresa.

- Si un trabajador siente un desafío como propio y capaz de superarlo, su rendimiento aumenta de manera progresiva, a pesar que el desafío ya no esté presente.

- Debido a la relación y manejo de conflictos entre los trabajadores y jefes o dueño de la empresa (en calidad de familia), el trato entre ellos influye enormemente en el rendimiento de sus labores, llegando en algunos casos de discusión, al abandono intempestivo de las labores, esto se pudo verificar de manera visual dentro de la empresa.

- El rendimiento de los trabajadores resultó ser afectada directamente por las dimensiones cooperación y estándar, todo esto en medida de la percepción de los trabajadores. En algunos casos se observó dentro de la organización, la falta de apoyo en el trabajo y de respeto a las reglas implícitas.

- La identidad o capacidad de poder identificarse con la empresa, es proporcional al compromiso que los mismos trabajadores brindan durante el desarrollo de sus tareas, ya que para ellos es una motivación, ser reconocidos como parte de la empresa.

\section{REFERENCIAS BIBLIOGRÁFICAS}

Al-Makhaita, H. M., Ahmed, S. A., \& Hafez, A. (2014). Job performance among nurses working in two different health care levels, Eastern Saudi Arabia: A comparative study. International Journal of Medical Science and Public Health, 3(7), 832837.

Borman, W. C., \& Motowidlo, S. M. (1993). Expanding the criterion domain to include elements of contextual performance. En N. Schmitt y W. C. Borman (Eds.), Personnel selection in organizations (pp. 71-98). San Francisco, Estados Unidos: Jossey-Bass. 
Brunet, L. (1987). El Clima de trabajo en las Organizaciones. Definiciones, diagnóstico y consecuencias. México DF. México: Trillas.

Delgado, Y., \& Adla, J. (2017). Clima y Compromiso Organizacional. Durango, México: Editorial Instituto Universitario Anglo Español.

Forehand, G. A., \& Gilmer, B. (1964). Environmental variations in studies of organisational behaviour. Psychological Bulletin, (62), 361-382.

Gabini, S. (2018). Potenciales predictores del rendimiento laboral. Buenos Aires, Argentina: Teseo.

Gutiérrez, E. (2001). Análisis descriptivo del clima organizacional antes y después de un proceso de rotación personal (tesis doctoral). Universidad Católica Andrés Bello, Caracas, Venezuela.

Halpin, A.W., \& Crofts, D.B. (1963). The organizational climate of schools. Chicago, Estados Unidos: Midwest Administration Center of the University of Chicago.

Huamaní, N. (2015). El clima organizacional y su influencia en el desempeño laboral del personal de conducción de trenes, del área de transportes del metro de Lima, la Línea 1 en el 2013 (tesis de pregrado). Universidad Nacional Tecnológica de Lima Sur, Villa el Salvador, Perú

Jahuira, L. (2015). Percepción del clima organizacional que tienen los profesionales de la salud en el Hospital de Apoyo Sandia II- 1, Puno 2015 (tesis de pregrado). Universidad Nacional del Altiplano, Puno, Puno.

Koopmans, L., Bernaards, C. M., Hildebrandt, V. H., Schaufeli, W. B., De Vet, H. C. W., \& Van Der Beek, A. J. (2011). Conceptual frameworks of individual work performance - A Systematic Review. Journal of Occupational and Environmental Medicine, 53(8), 856-866.
Koopmans, L., Bernaards, C. M., Hildebrandt, V. H., Lerner, D., De Vet, H. C., \& Van Der Beek, A. J. (2016). Cross-cultural adaptation of the Individual Work Performance Questionnaire. Work, 53(3), 609-619.

Kossen, S. (1995). Recursos Humanos en las organizaciones. México DF, México: Harla.

Litwin, G. H., \& Stringer, R. A. (1968). Motivation and organizational climate. Boston, Estados Unidos: Harvard Business School Press.

Murphy, K. R. (1990). Job performance and productivity. En K. R. Murphy y F. E. Saal (Eds). Psychology in organizations: Integrating science and practice (pp. 79-176). Hillsadle, Estados Unidos: Erlbaum.

Palacios, M. (2019). El clima organizacional y su relación con el desempeño laboral del personal administrativo de los distritos de Salud Pública de la provincia de Manabí Ecuador (tesis doctoral). Universidad Nacional Mayor de San Marcos, Lima, Perú.

Pelaes, O. (2010). Relación entre el clima organizacional y la satisfacción del cliente en una empresa de servicios telefónicos (tesis doctoral). Universidad Nacional Mayor de San Marcos, Lima, Perú.

Tapies, D. J. (2016). Lección inaugural del curso académico 2016/2017. Pamplona, España: Universidad de Navarra. 